\begin{tabular}{|c|l|}
\hline Title & TRIM59 interacts with ECSIT and negatively regulates NF-K B and IRF-3/-mediated signal pathways \\
\hline Author(s) & Kondo, Takeshi; W atanabe, Masashi; Hatakeyama, Shigetsugu \\
\hline Citation & $\begin{array}{l}\text { Biochemical and Biophysical Research Communications, 422(3), 501-507 } \\
\text { https://doi.org/40.1016j.bbrc.2012.05.028 }\end{array}$ \\
\hline Issue Date & 2012-06-08 \\
\hline Doc URL & http://hdl.handle.net/2115/49795 \\
\hline Type & article(author version) \\
\hline File Information & BBRC422-3_501-507.pdf \\
\hline
\end{tabular}

Instructions for use 


\section{TRIM59 interacts with ECSIT and negatively regulates NF- $\kappa$ B and IRF-3/7-mediated signal pathways}

Takeshi Kondo ${ }^{\text {a }}$, Masashi Watanabe ${ }^{\text {a }}$ and Shigetsugu Hatakeyama ${ }^{\text {a,* }}$

a Department of Biochemistry, Hokkaido University Graduate School of Medicine, Sapporo, Hokkaido 060-8638, Japan

* Corresponding author: Shigetsugu Hatakeyama, Department of Biochemistry, Hokkaido University Graduate School of Medicine, Kita 15, Nishi 7, Kita-ku, Sapporo, Hokkaido 060-8638, Japan. Tel.: +81 11706 5899; fax: +81 117065169 . E-mail address: hatas@med.hokudai.ac.jp (S. Hatakeyama)

Key words: TRIM59, ECSIT, IPS-1, NF-кB, IRF 


\section{Abstract}

Innate immune responses are triggered by pathogen-associated molecular patterns (PAMPs) through pattern recognition receptors (PRRs) and then activate intracellular signaling pathways including NF- $\mathrm{KB}$ and interferon regulatory factors. Recently, it has been reported that tripartite motif (TRIM) proteins function as crucial regulators via ubiquitin-mediated modifications for these signaling pathways. In this study, we showed that one of the TRIM family ubiquitin ligases, TRIM59, interacts with ECSIT as an adaptor protein required for the TLR-mediated transduction pathway. Luciferase reporter assays using reporter plasmids including $\mathrm{NF}-\kappa \mathrm{B}$ responsive element, interferon $\beta$ (IFN- $\beta$ ) promoter and interferon-sensitive response element (ISRE) showed that overexpression of TRIM59 repressed their transcriptional activities, whereas knockdown of TRIM59 enhanced their transcriptional activities. Furthermore, TRIM59 inhibited phosphorylation and dimerization of IRF3 and IRF7, suggesting that TRIM59 negatively regulates upstream kinases for IRFs. These findings indicate that TRIM59 may serve as a multifunctional regulator for innate immune signaling pathways. 


\section{Introduction}

Innate immune responses are triggered by the engagement of Toll-like receptors (TLRs) or other pattern-recognition receptors (PRRs) by various pathogen components, called pathogen-associated molecular patterns (PAMPs) [1]. These receptors sense PAMPs and trigger intracellular signaling pathways that activate NF- $\kappa \mathrm{B}, \mathrm{IRF} 3$ and IRF7. RIG-I-like receptors (RLRs), such as RIG-I and MDA5, recognize viral RNA and transmit signals to the downstream mitochondrion-located adaptor protein IPS-1 (also known as MAVS, VISA, and Cardif) [2]. IPS-1 then activates IKK-protein-kinase family members.

Tripartite motif-containing (TRIM) superfamily proteins have a TRIM or RBCC motif consisting a RING domain, one or two B-box domains and an associated coiled-coil domain. TRIM proteins are involved in a various biological processes $[3,4]$. TRIM25, also known as estrogen-responsive finger protein (EFP), induces type I IFN production and $\mathrm{NF}-\kappa \mathrm{B}$ activity through the conjugation of a lysine 63-linked polyubiquitin chain to RIG-I [5]. Another study showed that TRIM25 also has an E3 ligase activity for ISG15 conjugation [6]. These findings suggest that TRIM25 positively and negatively regulates type I IFN production. TRIM59 and TRIM13 have a putative carboxy-terminal transmembrane domain. TRIM13 has an E3 ubiquitin ligase activity and localizes to the endoplasmic reticulum (ER) by its transmembrane domain [7]. TRIM13 interacted with several proteins required for ER-associated degradation (ERAD), including VCP [7]. It has been reported that knockdown of TRIM59 in human 
prostate cancer cells causes cell-cycle arrest and cell growth retardation and that poorly differentiated carcinomas developed in prostate tissue-specific TRIM59 transgenic mice [8].

In this study, to elucidate the molecular function of TRIM59, we performed yeast two-hybrid screening using TRIM59 as bait and identified a signal adaptor protein, evolutionarily conserved signaling intermediate in Toll pathways (ECSIT), as a TRIM59-interacting protein. We found that ECSIT is involved in RLR signaling and that TRIM59 may suppress RLR-induced activation of IRFs and NF- $\kappa \mathrm{B}$ via interaction with ECSIT. These findings indicate that TRIM59 functions as a novel regulator for innate immune signaling. 


\section{Materials and methods}

\subsection{Reagents}

The following commercial antibodies were used: anti-FLAG (M2, Sigma, Louis, MO), $\beta$-actin (AC15, Sigma), anti-HA (HA.11, Covance Research Products, Berkeley, CA), anti-HA (Y11, Santa Cruz, Santa Cruz, CA), anti-Myc (N262, Santa Cruz, and 9E10, Covance), anti-ubiquitin (FK2, Nippon Bio-Test Laboratories Inc, Tokyo, Japan and P4D1, Santa Cruz), and anti-Hsp90 (BD, Franklin Lakes, NJ). Rabbit anti-TRIM59 antiserum was raised against recombinant mouse truncated TRIM59 (1-280 amino acid residues) (Hokkaido System Science Co. Ltd., Sapporo, Japan). LPS (E. coli 055:B5) and poly(I:C) were purchased from Sigma.

\subsection{Cell culture}

HEK293, HEK293T, and HeLa cell lines were cultured under an atmosphere of 5\% $\mathrm{CO}_{2}$ at $37^{\circ} \mathrm{C}$ in Dulbecco's modified Eagle's medium (Sigma) supplemented with $10 \%$ fetal bovine serum (FBS) (Gibco BRL, Paisley, UK).

\subsection{Cloning of cDNAs and plasmid construction}

Mouse TRIM59 was amplified by PCR from a mouse E17 cDNA library (Clontech 
Laboratories, Inc. Mountain View, CA) with KOD PLUS (Toyobo, Osaka, Japan) using the following PCR primers:; 5'-GAAATGCACAATTTTGAGGAGGAG-3' for TRIM59 forward primer and 5'-TTTTCAACGAGAAACTATTTTCCA-3' for TRIM59 reverse primer. The amplified fragments were subcloned into pBluescript II SK+ (Stratagene, La Jolla, CA). The resulting fragments containing TRIM59 cDNA were subcloned into pCR vector (Invitrogen, Carlsbad, CA) with a FLAG-tag or HA-tag, pBTM116 (Clontech), pGEX-6P vector (Amersham Bioscience) and pMX-puro, which was obtained from Dr. T. Kitamura (University of Tokyo). TRIM59 cDNA lacking the RING domain (TRIM59( $\triangle \mathrm{R})$ ) was amplified by PCR and subcloned into pCR and pMX-puro vector. A truncated mutant lacking the transmembrane domain (TRIM59- $\Delta$ TM) was cut out by using XhoI and inserted into expression vectors.

\subsection{Retroviral expression system}

Complementary DNAs encoding wild-type TRIM59 or TRIM59( $\Delta \mathrm{R})$ containing HA-tags at their N-termini were subcloned into pMX-puro provided by Dr. Kitamura. The resulting vectors were used for transfection into Plat E cells and thereby recombinant retroviruses were generated [9]. HeLa cells expressing mCAT1 were infected with the recombinant retroviruses and selected in a medium containing puromycin $(8 \mu \mathrm{g} / \mathrm{ml})$ [10].

\subsection{RNA interference}


Hairpin sequences specific for human TRIM59 mRNAs that corresponded to the following regions with the U6 promoter sequence were subcloned into pMX-puro vector: shTRIM59-1 (5'-AAGAGGGCCAATTAAATCAAA-3') and shTRIM59-2 (5'-AAGAATGGAGCAGAACAGAAA-3'). Hairpin sequences specific for enhanced green fluorescent protein (GFP, Clontech) mRNA were used as controls.

\subsection{Luciferase reporter assay}

To construct the reporter plasmid IFN- $\beta$ luc, the murine IFN- $\beta$ promoter region was amplified by PCR using sense 5'-ATGACAGAGGAAAACTGAAAG-3' and antisense 5'-GCTGCAGTGAGAATGATCTTC-3' primers and subcloned into pGL4.20 vector (Promega, Madison, WI). HEK293 or HeLa cells were inoculated into a 24-well dish at

$5 \times 10^{4}$ cells $/ 500 \mu \mathrm{l}$ of cell culture medium and were transiently transfected using FuGENE HD (Roche, Mannheim, Germany) with expression vectors, reporter plasmids, and internal control plasmid coding Renilla luciferase. At $24 \mathrm{~h}$ after transfection, cells were harvested and lysed in $100 \mu \mathrm{l}$ of cell culture lysis reagent (Promega), and then luciferase activity was measured using $5 \mu \mathrm{l}$ of lysate and $25 \mu \mathrm{l}$ of luciferase assay substrate (Promega). The luminescence was quantified using a Dual-Luciferase Reporter Assay System (Promega) with a luminometer (Promega). A reporter plasmid containing ISRE was kindly provided by Dr. Hirano (Osaka University) [11]. A reporter plasmid containing the NF-אB responsive sequence was described previously [12]. 


\subsection{Yeast two-hybrid screening}

Complementary DNA encoding amino acids 1-280 of TRIM59 was fused in-frame to the nucleotide sequence for the LexA domain (BD) in the yeast two-hybrid vector pBTM116. For yeast two-hybrid screening, the yeast strain L40 (Invitrogen) was transformed with an T-cell Matchmaker cDNA library (Clontech) using the lithium acetate method.

\subsection{Transfection, immunoprecipitation and immunoblot analysis}

HEK293T cells were transfected by the calcium phosphate method. Thirty-six hours after transfection, cells were lysed in a solution containing $50 \mathrm{mM}$ Tris- $\mathrm{HCl}(\mathrm{pH}$ 7.4), $150 \mathrm{mM} \mathrm{NaCl}, \quad 1 \% \quad$ Triton-X 100, leupeptin $(10 \mu \mathrm{g} / \mathrm{ml}), \quad 1 \quad \mathrm{mM}$ phenylmethylsulfonyl fluoride, $400 \mu \mathrm{M} \mathrm{Na}_{3} \mathrm{VO}_{4}, 400 \mu \mathrm{M}$ EDTA, $10 \mathrm{mM} \mathrm{NaF}$, and 10 $\mathrm{mM}$ sodium pyrophosphate. The cell lysates were centrifuged at $15,000 \mathrm{~g}$ for $10 \mathrm{~min}$ at $4^{\circ} \mathrm{C}$, and the resulting supernatant was incubated with antibodies for $1 \mathrm{~h}$ at $4^{\circ} \mathrm{C}$. Protein A-Sepharose (Amersham Biosciences) was added to the mixture and then rotated for $1 \mathrm{~h}$ at $4^{\circ} \mathrm{C}$. The resin was washed five times with ice-cold lysis buffer, and then boiled in SDS sample buffer. Immunoblot analysis was performed with the primary antibodies, horseradish peroxidase-conjugated antibodies to mouse or rabbit immunoglobulin $\mathrm{G}$ (1:15,000 dilution, Promega) and an enhanced chemiluminescence system (ECL, 
Amersham Pharmacia).

\subsection{Immunofluorescence staining}

Cells were fixed with $4 \%$ formaldehyde in PBS for $15 \mathrm{~min}$ at room temperature and incubated with indicated antibodies at a concentration of $1 \mu \mathrm{g} / \mathrm{ml}$ in PBS containing $0.1 \% \mathrm{BSA}$ and $0.1 \%$ saponin for $1 \mathrm{~h}$ at room temperature, followed by incubation with anti-mouse or rabbit Alexa 488 or 546 antibodies (Invitrogen) at a dilution of 1:500 for $1 \mathrm{~h}$ at room temperature. Cells were then photographed with a confocal microscope MRC-1024 (Bio-Rad).

\subsection{Native-PAGE}

Cell lysates were prepared in lysis buffer containing $50 \mathrm{mM}$ Tris- $\mathrm{HCl}(\mathrm{pH} 8.0), 1 \%$ $\mathrm{NP} 40,150 \mathrm{mM} \mathrm{NaCl}$. The $8 \%$ native polyacrylamide gel was pre-run at $40 \mathrm{~mA}$ for 30 min, loaded with samples, and run at $25 \mathrm{~mA}$ for $50 \mathrm{~min}$. The upper chamber buffer contained $25 \mathrm{mM}$ Tris- $\mathrm{HCl}(\mathrm{pH} 8.4), 192 \mathrm{mM}$ glycine, and $0.2 \%$ sodium deoxycholate and the lower chamber buffer was Tris- $\mathrm{HCl}(\mathrm{pH} 8.4)$ and $192 \mathrm{mM}$ glycine. The proteins in the PAGE gel were denatured in $20 \mathrm{mM}$ Tris ( $\mathrm{pH} 8.4$ ), $150 \mathrm{mM}$ glycine, and $0.1 \%$ SDS for $10 \mathrm{~min}$ and blotted onto an Immobilon-P membrane (Millipore Corporation, Bedford, MA). 


\subsection{Statistical analysis}

Student's $t$-test was used to determine the statistical significance of experimental data. 


\section{Results}

\subsection{TRIM59 is localized in the ER}

Based on the protein structure, TRIM59 and TRIM13 have putative transmembrane domains in the carboxy-terminal region. Actually, TRIM13 has been reported to be a transmembrane protein in the ER and to be involved in the ERAD [7]. Although TRIM59 has been reported to have an oncogenic activity, its subcellular localization has not been clarified [8].

To investigate the localization of TRIM59, we generated and transfected an expression vector encoding wild-type TRIM59 (TRIM59(WT)) or a deletion mutant of TRIM59 lacking a transmembrane domain at the carboxy-terminus (TRIM59( $\Delta \mathrm{TM})$ ) with an expression vector encoding DsRed-ER (Fig. 1A and B). Immunofluorescence analysis using confocal microscopy showed that TRIM59(WT) localizes to the ER, whereas TRIM59( $\Delta \mathrm{TM})$ localizes to the cytoplasm, not to the ER (Fig. 1B). The lack of a RING domain in TRIM59 (TRIM59( $\Delta \mathrm{R})$ ) did not affect its localization.

\subsection{Identification of ECSIT as a novel TRIM59-interacting protein}

To investigate the function of TRIM59, we tried to identify TRIM59-interacting

proteins by yeast two-hybrid screens. Using TRIM59 as bait, we screened $6 \times 10^{5}$ independent clones from mouse T cell cDNA libraries and obtained $21 \beta$-gal-positive 
clones. Sequence analysis of DNA fragments from those clones demonstrated that one of the fragments encoded ECSIT lacking amino-terminal 27 amino acids.

To confirm that TRIM59 interacts with ECSIT in mammalian cells, we expressed HA-tagged TRIM59 together with Myc-tagged ECSIT and performed a coimmunoprecipitation assay. The assay showed that HA-tagged TRIM59 is co-precipitated with ECSIT, indicating that TRIM59 specifically interacts with ECSIT in mammalian cells (Fig. 1C). Next, to examine which region of TRIM59 is required for the interaction with ECSIT, coimmunoprecipitation experiments were performed using TRIM59 deletion mutants (Fig. 1A and D). An in vivo binding assay showed that TRIM59( $\Delta \mathrm{R})$ and TRIM59(CC) weakly interacted with ECSIT, whereas TRIM59(B) and TRIM59( $\triangle \mathrm{TM})$ strongly interacted with ECSIT (Fig. 1D). These findings suggest that the B-box and RING domains of TRIM59 are important for interaction with ECSIT.

We verified by in vitro ubiquitination assay that TRIM59 has an E3 ligase activity (data not shown). However, overexpression of TRIM59(WT) did not increase ubiquitination levels of Myc-tagged ECSIT in HEK293T cells (data not shown), suggesting that TRIM59 interacts with ECSIT but that ECSIT is not a direct target for ubiquitination by TRIM59.

\subsection{ECSIT interacts with IPS-1 and positively regulates RLR-mediated signaling}

A recent study on RLR signaling showed that ECSIT-interacting proteins, TRAF6 and MEKK1, are important as downstreams of IPS-1 localized at mitochondria [13] and 
that ECSIT has a amino-terminal targeting sequence to localize to mitochondria [14]. Therefore, we examined whether ECSIT interacts with IPS-1. Immunofluorescence analysis showed that ECSIT and IPS-1 are partially colocalized at mitochondria (Fig. 2A). To verify the interaction between IPS-1 and ECSIT, we performed an in vivo binding assay. FLAG-tagged IPS-1 and Myc-tagged ECSIT were expressed in HEK293T cells and coimmunoprecipitation experiments were performed. Myc-tagged ECSIT was coprecipitated with IPS-1, suggesting that TRIM59 regulates IPS-1 function via ECSIT (Fig. 2B).

To examine whether ECSIT affects IFN- $\beta$ promoter-mediated transcription by IPS-1 overexpression, we performed a luciferase assay with a reporter plasmid in which the IFN- $\beta$ promoter region was inserted. Overexpression of IPS-1 induced activation of the IFN- $\beta$ promoter, and coexpression of ECSIT further promoted its activation, whereas a dominant-negative form of $\operatorname{ECSIT~}(\operatorname{ECSIT}(\Delta N))$ lost the transcriptional activation [15] (Fig. 2C and D). These findings indicate that ECSIT interacts with IPS-1 at mitochondria and may positively regulate RLR-mediated signaling.

3.4. TRIM59 overexpression suppresses activation of NF- $\mathrm{kB}$ and IFN- $\beta$ promoter

Since ECSIT is involved in RLR-signaling, we examined whether TRIM59 affects virus-induced type I IFN signaling. We generated HeLa cells that stably expressed HA-tagged TRIM59 (Fig. 3A) and transiently transfected a reporter plasmid with the IFN- $\beta$ promoter. Luciferase reporter assays indicated that TRIM59 inhibited 
poly(I:C)-induced activation of the IFN- $\beta$ promoter in HeLa cells (Fig. 3B). Next, we examined whether TRIM59 functions downstream of IPS-1. TRIM59 overexpression inhibited IPS-1-induced activation of the ISRE or NF- $\kappa \mathrm{B}$ promoter in HEK293T cells, whereas a catalytic inactive mutant of TRIM59 (TRIM59( $\Delta \mathrm{R}))$ lost its ability to inhibit IPS-1-induced activation (Fig. 3C and D).

We then examined whether TRIM59 regulates IPS-1-mediated dimer formation of IRF3 and IRF7, which is observed after phosphorylation by upstream kinases. Immunoblot analysis showed that IPS-1-induced dimerization of IRF3 and IRF7 is impaired by TRIM59 overexpression. TRIM59 also inhibited the phosphorylation of IRF3 and IRF7, which induces dimerization, suggesting that TRIM59 negatively regulates kinases for IRF3/7 (IKKe/TBK1) or their upstream signal (Fig. 3E). These findings indicate that TRIM59 inhibits signaling for induction of IFN- $\beta$ and that this inhibitory function requires E3 ubiquitin ligase activity of TRIM59.

\subsection{Knockdown of TRIM59 promotes activation of $N F-\kappa B$ and IFN- $\beta$ promoter}

To confirm the physiological role of TRIM59 in the NF- $\kappa B$ signal, ISRE and IFN- $\beta$ promoter, we knocked down endogenous TRIM59 in HeLa cells. The shRNA treatment resulted in significant silencing of TRIM59 at the protein level in HeLa cells (Fig. 4A). To examine whether endogenous TRIM59 regulates the NF- $\kappa B$ signal, ISRE and IFN- $\beta$ promoter, the established cell lines were used for luciferase reporter assays. Luciferase assays showed that knockdown of TRIM59 also promotes poly(I:C)-induced activation 
of the IFN- $\beta$ promoter in HeLa cells (Fig. 4B). Furthermore, luciferase assays showed that knockdown of TRIM59 promotes IPS-1-induced activation of NF-kB- and ISRE-mediated transcription in HeLa cells (Fig. 4C and D). These findings suggest that TRIM59 is a physiological regulator for IRF and NF- $\mathrm{B}$ activation and IFN- $\beta$ induction. 


\section{Discussion}

In this study, we showed that TRIM59 interacts with ECSIT as an adaptor protein required for the TLR pathway and that ECSIT functions as a positive regulator in the IFN- $\beta$ promoter. Overexpression of TRIM59 repressed their transcriptional activities in the NF- $\kappa \mathrm{B}$ responsive element, IFN- $\beta$ or ISRE promoter, whereas knockdown of TRIM59 enhanced their transcriptional activities. These findings suggest that TRIM59 may serve as a negative regulator for innate immune signaling pathways.

Recently it has been reported that some membrane proteins including IPS-1 and STING play a key role for adequate control of innate immune signaling [2, 16-18]. Since we identified ECSIT as a novel TRIM59-binding protein and showed that ECSIT is required for the TLR-mediated signal transduction pathway, we hypothesized that TRIM59 is involved in the RLR pathway with ECSIT. We showed that that ECSIT interacts with IPS-1 as an important transducer for innate immune signaling and promotes activation of the IFN- $\beta$ promoter. Furthermore, TRIM59 overexpression inhibited activation of transcription factors that are mediated via IPS-1, whereas knockdown of TRIM59 had the opposite effects. These results indicated that endogenous TRIM59 acts as a negative regulator for IRFs/NF- $\kappa \mathrm{B}$ activation and IFN- $\beta$ induction. Since TRIM59 inhibited phosphorylation and dimerization of IRF3 and IRF7, TRIM59 negatively regulates kinases for IRF3/7 (IKKe/TBK1) or their upstream signal. Luciferase reporter assays showed that the RING domain of TRIM59 is required for inhibition of the IFN- $\beta$ promoter. TRIM59 interacts with ECSIT as a positive regulator 
in the IFN- $\beta$ promoter in collaboration with IPS-1. Therefore, we hypothesized that TRIM59 mediates ubiquitination and degradation of ECSIT. However, we found by a ubiquitination assay and a protein stability assay that TRIM59 does not facilitate ubiquitination or degradation of ECSIT. Recently, it has been reported that TLR signals involve translocation of TRAF6 to mitochondria and engagement of ECSIT on the mitochondrial surface and that TRAF6 leads to ECSIT ubiquitination and enrichment at the mitochondrial periphery, resulting in increased mitochondrial and cellular ROS generation [19]. Hence, it is important to clarify molecular linkages of TRIM59 and TRAF6 to regulate ECSIT in the TLR signaling pathway.

Previous studies have shown that many TRIM proteins are induced in response to interferons and that TRIM proteins are important components of resistance to pathogens and are involved in many biological processes [20]. TRIM5 is involved in pathogen recognition and regulation of transcriptional pathways in host defense. TRIM5 $\alpha$ inhibits infection of HIV-1 and other retroviruses upon entry into the cell by engaging the viral capsid and inducing premature uncoating [21]. Moreover, it has been reported that interferon signaling and $\mathrm{NF}-\kappa \mathrm{B}$ signaling are regulated by TRIM proteins including TRIM8, TRIM21, TRIM25, TRIM27, TRIM30 and TRIM56 [5, 22-27]. Recently, it has been reported that TRIM56 interacts with STING and targets it for lysine 63-linked ubiquitination, followed by induction of STING dimerization and activation of the IFN- $\beta$ promoter [27]. Previous studies showed that a transmembrane TRIM protein, TRIM13, is localized in the ER and regulates ER-stress induced autophagy [28]. Our results showed that TRIM59 is also localized in the ER via the carboxy-terminal 
transmembrane domain. Taken together, since membrane proteins such as IPS-1 and STING control innate immune signaling, TRIM59 may also function as an important regulator in collaboration with these molecules for these signaling pathways. Detailed studies including genetic analysis using TRIM59 knockout mice are needed to clarify the physiological roles of TRIM59 in RLR signaling. Further functional analysis of TRIM may lead to therapeutic benefits not only for suppression of infectious diseases but also for inflammation. 


\section{Acknowledgments}

We would like to thank Drs. Toshio Kitamura, Kentaro Hanada and Toshio Hirano for the plasmids and cell lines and Ms. Yuri Soida for help in preparing the manuscript. The work was supported in part by KAKENHI (18076001 and 21390087) from the Ministry of Education, Culture, Sports, Science and Technology in Japan, The Sumitomo Foundation and The Suhara Foundation (to S. Hatakeyama). 


\section{References}

[1] S. Akira, S. Uematsu, O. Takeuchi, Pathogen recognition and innate immunity, Cell 124 (2006) 783-801.

[2] S.M. Belgnaoui, S. Paz, J. Hiscott, Orchestrating the interferon antiviral response through the mitochondrial antiviral signaling (MAVS) adapter, Curr. Opin. Immunol. 23 (2011) 564-572.

[3] S. Hatakeyama, TRIM proteins and cancer, Nat. Rev. Cancer 11 (2011) 792-804.

[4] K. Ozato, D.M. Shin, T.H. Chang, H.C. Morse, 3rd, TRIM family proteins and their emerging roles in innate immunity, Nat. Rev. Immunol. 8 (2008) 849-860.

[5] M.U. Gack, Y.C. Shin, C.H. Joo, T. Urano, C. Liang, L. Sun, O. Takeuchi, S. Akira, Z. Chen, S. Inoue, J.U. Jung, TRIM25 RING-finger E3 ubiquitin ligase is essential for RIG-I-mediated antiviral activity, Nature 446 (2007) 916-920.

[6] W. Zou, D.E. Zhang, The interferon-inducible ubiquitin-protein isopeptide ligase (E3) EFP also functions as an ISG15 E3 ligase, J. Biol. Chem. 281 (2006) 3989-3994.

[7] M. Lerner, M. Corcoran, D. Cepeda, M.L. Nielsen, R. Zubarev, F. Ponten, M. Uhlen, S. Hober, D. Grander, O. Sangfelt, The RBCC gene RFP2 (Leu5) encodes a novel transmembrane E3 ubiquitin ligase involved in ERAD, Mol. Biol. Cell 18 (2007) 1670-1682.

[8] F. Valiyeva, F. Jiang, A. Elmaadawi, M. Moussa, S.P. Yee, L. Raptis, J.I. Izawa, B.B. Yang, N.M. Greenberg, F. Wang, J.W. Xuan, Characterization of the oncogenic 
activity of the novel TRIM59 gene in mouse cancer models, Mol. Cancer Ther. 10 (2011) 1229-1240.

[9] T. Kitamura, New experimental approaches in retrovirus-mediated expression screening, Int. J. Hematol. 67 (1998) 351-359.

[10] K. Hanada, K. Kumagai, S. Yasuda, Y. Miura, M. Kawano, M. Fukasawa, M. Nishijima, Molecular machinery for non-vesicular trafficking of ceramide, Nature 426 (2003) 803-809.

[11] K. Nakajima, Y. Yamanaka, K. Nakae, H. Kojima, M. Ichiba, N. Kiuchi, T. Kitaoka, T. Fukada, M. Hibi, T. Hirano, A central role for Stat3 in IL-6-induced regulation of growth and differentiation in M1 leukemia cells, EMBO J. 15 (1996) 3651-3658.

[12] M. Matsuda, T. Tsukiyama, M. Bohgaki, K. Nonomura, S. Hatakeyama, Establishment of a newly improved detection system for NF-kappaB activity, Immunol. Lett. 109 (2007) 175-181.

[13] R. Yoshida, G. Takaesu, H. Yoshida, F. Okamoto, T. Yoshioka, Y. Choi, S. Akira, T. Kawai, A. Yoshimura, T. Kobayashi, TRAF6 and MEKK1 play a pivotal role in the RIG-I-like helicase antiviral pathway, J. Biol. Chem. 283 (2008) 36211-36220.

[14] R.O. Vogel, R.J. Janssen, M.A. van den Brand, C.E. Dieteren, S. Verkaart, W.J. Koopman, P.H. Willems, W. Pluk, L.P. van den Heuvel, J.A. Smeitink, L.G. Nijtmans, Cytosolic signaling protein Ecsit also localizes to mitochondria where it interacts with chaperone NDUFAF1 and functions in complex I assembly, 
Genes Dev. 21 (2007) 615-624.

[15] E. Kopp, R. Medzhitov, J. Carothers, C. Xiao, I. Douglas, C.A. Janeway, S. Ghosh, ECSIT is an evolutionarily conserved intermediate in the Toll/IL-1 signal transduction pathway, Genes Dev. 13 (1999) 2059-2071.

[16] H. Ishikawa, G.N. Barber, STING is an endoplasmic reticulum adaptor that facilitates innate immune signalling, Nature 455 (2008) 674-678.

[17] B. Zhong, Y. Yang, S. Li, Y.Y. Wang, Y. Li, F. Diao, C. Lei, X. He, L. Zhang, P. Tien, H.B. Shu, The adaptor protein MITA links virus-sensing receptors to IRF3 transcription factor activation, Immunity 29 (2008) 538-550.

[18] W. Sun, Y. Li, L. Chen, H. Chen, F. You, X. Zhou, Y. Zhou, Z. Zhai, D. Chen, Z. Jiang, ERIS, an endoplasmic reticulum IFN stimulator, activates innate immune signaling through dimerization, Proc. Natl.. Acad. Sci. USA 106 (2009) 8653-8658.

[19] A.P. West, I.E. Brodsky, C. Rahner, D.K. Woo, H. Erdjument-Bromage, P. Tempst, M.C. Walsh, Y. Choi, G.S. Shadel, S. Ghosh, TLR signalling augments macrophage bactericidal activity through mitochondrial ROS, Nature 472 (2011) 476-480.

[20] P.D. Uchil, B.D. Quinlan, W.T. Chan, J.M. Luna, W. Mothes, TRIM E3 ligases interfere with early and late stages of the retroviral life cycle, PLoS Pathog. 4 (2008) e16.

[21] T. Pertel, S. Hausmann, D. Morger, S. Zuger, J. Guerra, J. Lascano, C. Reinhard, F.A. Santoni, P.D. Uchil, L. Chatel, A. Bisiaux, M.L. Albert, C. 
Strambio-De-Castillia, W. Mothes, M. Pizzato, M.G. Grutter, J. Luban, TRIM5 is an innate immune sensor for the retrovirus capsid lattice, Nature 472 (2011) 361-365.

[22] Q. Li, J. Yan, A.P. Mao, C. Li, Y. Ran, H.B. Shu, Y.Y. Wang, Tripartite motif 8 (TRIM8) modulates TNFalpha- and IL-1beta-triggered NF-kappaB activation by targeting TAK1 for K63-linked polyubiquitination, Proc. Natl.. Acad. Sci. USA 108 (2011) 19341-19346.

[23] R. Higgs, J. Ni Gabhann, N. Ben Larbi, E.P. Breen, K.A. Fitzgerald, C.A. Jefferies, The E3 ubiquitin ligase Ro52 negatively regulates IFN-beta production post-pathogen recognition by polyubiquitin-mediated degradation of IRF3, J. Immunol. 181 (2008) 1780-1786.

[24] K. Yang, H.X. Shi, X.Y. Liu, Y.F. Shan, B. Wei, S. Chen, C. Wang, TRIM21 is essential to sustain IFN regulatory factor 3 activation during antiviral response, J. Immunol. 182 (2009) 3782-3792.

[25] J. Zha, K.J. Han, L.G. Xu, W. He, Q. Zhou, D. Chen, Z. Zhai, H.B. Shu, The Ret finger protein inhibits signaling mediated by the noncanonical and canonical IkappaB kinase family members, J. Immunol. 176 (2006) 1072-1080.

[26] M. Shi, W. Deng, E. Bi, K. Mao, Y. Ji, G. Lin, X. Wu, Z. Tao, Z. Li, X. Cai, S. Sun, C. Xiang, B. Sun, TRIM30 alpha negatively regulates TLR-mediated NF-kappa $\mathrm{B}$ activation by targeting TAB2 and TAB3 for degradation, Nat. Immunol. 9 (2008) 369-377.

[27] T. Tsuchida, J. Zou, T. Saitoh, H. Kumar, T. Abe, Y. Matsuura, T. Kawai, S. Akira, 
The ubiquitin ligase TRIM56 regulates innate immune responses to intracellular double-stranded DNA, Immunity 33 (2010) 765-776.

[28] D. Tomar, R. Singh, A.K. Singh, C.D. Pandya, TRIM13 regulates ER stress induced autophagy and clonogenic ability of the cells, Biochim. Biophys. Acta 1823 (2012) 316-326. 


\section{Figure legends}

Fig. 1. TRIM59 is localized in the ER and interacts with ECSIT. (A) Schematic representation of full-length TRIM59 and its deletion mutants is shown. Protein motifs are indicated: RING, Ring-finger domain; B-box, B-box domain; C-coil, coiled-coil domain; TM, transmembrane domain. (B) Confocal microscopic analysis of TRIM59. HeLa cells were cotransfected with HA-tagged TRIM59 mutants and DsRed-ER. Twenty-four hours after transfection, cells were fixed with $4 \%$ paraformaldehyde and stained with anti-HA antibody and Hoechst33258. Scale bars, $20 \mu \mathrm{m}$. (C) In vivo binding assay between TRIM59 and ECSIT. Expression vectors encoding HA-tagged TRIM59 and Myc-tagged ECSIT were transfected into HEK293T cells. Twenty-four hours after transfection, MG132 $(2 \mu \mathrm{M})$ was added to the culture medium and incubated for $18 \mathrm{~h}$. Cell lysates were immunoprecipitated (IP) with anti-HA or anti-Myc antibody and immunoblotted (IB) with anti-HA and anti-Myc antibodies. (D) The B-box and RING domains of TRIM59 are required for binding to ECSIT. HEK293T cells were transfected with vectors encoding Myc-tagged ECSIT and either HA-tagged wild type (WT) or deletion mutants of TRIM59. Cell lysates were immunoprecipitated with anti-HA antibody and then immunoblotted with anti-Myc antibody.

Fig. 2. ECSIT enhances IPS-1-mediated IFN- $\beta$ promoter activation. (A) ECSIT is partially colocalized with IPS-1. HeLa cells were co-transfected with expression vectors encoding ECSIT-Myc and HA-IPS-1. Twenty-four hours after transfection, cells were 
fixed with $4 \%$ paraformaldehyde, stained with the indicated antibodies, and subjected to confocal microscopy. Scale bars, $20 \mu \mathrm{m}$. (B) ECSIT interacts with IPS-1. Expression vectors encoding Myc-tagged ECSIT and FLAG-tagged IPS-1 were transfected into HEK293T cells. Thirty-six hours after transfection, cells were harvested and lysed. Cell lysates (input) were immunoprecipitated (IP) with anti-HA or anti-Myc antibody and immunoblotted (IB) with anti-HA and anti-Myc antibodies. (C) Schematic representation of ECSIT and its mutant (top) and promotion of IPS-1-induced activation of the IFN- $\beta$ promoter by ECSIT (bottom). (D) ECSIT enhances IFN- $\beta$ promoter activation. HEK293 cells were transfected with an IFN- $\beta$ promoter reporter plasmid and the indicated expression plasmids. Luciferase assays were performed $24 \mathrm{hr}$ after transfection.

Fig. 3. TRIM59 overexpression inhibits innate immune signaling pathways. (A) Establishment of HeLa cells stably expressing FLAG-tagged TRIM59 by a retroviral expression system. The cell lines were checked by immunoblot analysis using anti-HA antibody. (B) TRIM59 represses poly(I:C)-induced activation of the IFN- $\beta$ promoter. HeLa cells that stably express HA-TRIM59 were transfected with an IFN- $\beta$ promoter reporter. Twenty hours after transfection, cells were further transfected with Poly(I:C). Six hours after Poly(I:C) transfection, luciferase assays were performed. (C) TRIM59 inhibits IPS-1-induced NF-kB-mediated transcriptional activation. HEK293 cells were transfected with an NF- $\kappa \mathrm{B}$ reporter and the indicated expression plasmids. Twenty-four hours after transfection, luciferase assays were performed. (D) TRIM59 inhibits 
IPS-1-induced ISRE activation. Experiments were performed as shown in (C) except that an ISRE reporter plasmid was used. (E) TRIM59 inhibits IPS-1-induced IRF3/IRF7 dimerization. HEK293T cells were transfected with the indicated plasmids. Thirty-six hours after transfection, cells were harvested and cell lysates were separated by Native-PAGE (upper panel) or SDS-PAGE (bottom five panels) and analyzed by immunoblotting with the indicated antibodies.

Fig. 4. Knockdown of TRIM59 enhances innate immune signaling pathways. (A) Knockdown of TRIM59 in HeLa cells. Short hairpin RNAs targeting TRIM59 (shTRIM59-1 and shTRIM59-2) or scrambled shRNA as a negative control (Mock) were introduced into HeLa cells by a retroviral expression system. Cell lysates were subjected to immunoblotting using anti-TRIM59 or anti-Hsp90 antibody. (B) Effect of TRIM59 shRNA on poly(I:C)-induced IFN- $\beta$ promoter activation. HeLa cells in which TRIM59 was knocked down were transfected with an IFN- $\beta$ promoter reporter. Twenty hours after transfection, cells were further transfected with Poly(I:C). Six hours after Poly(I:C) transfection, luciferase assays were performed. (C) Effect of TRIM59 RNAi

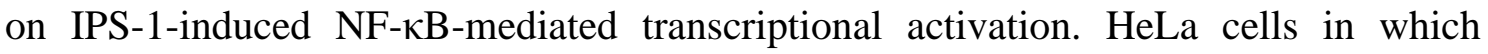
TRIM59 was knocked down were transfected with an NF- $\mathrm{B}$ reporter. Twenty-four hours after transfection, luciferase assays were performed. (D) Effect of TRIM59 RNAi on IPS-1-induced ISRE promoter activation. HeLa cells in which TRIM59 was knocked down were transfected with an ISRE promoter reporter. Twenty-four hours after transfection, luciferase assays were performed. 
A

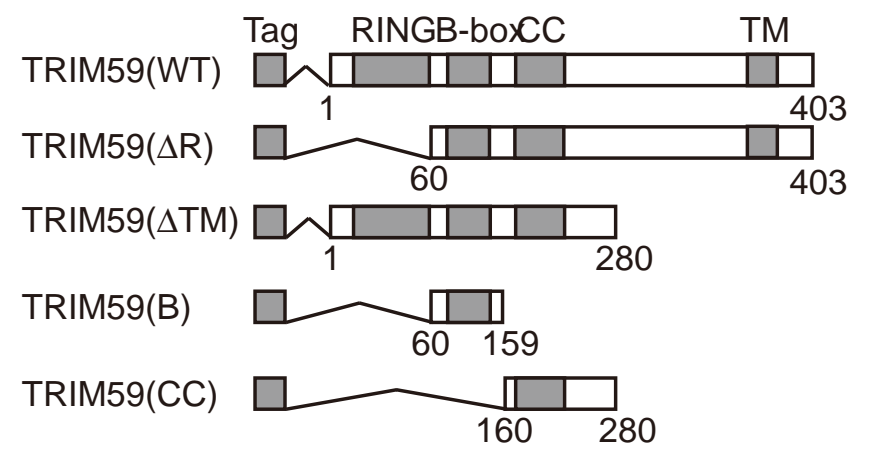

C

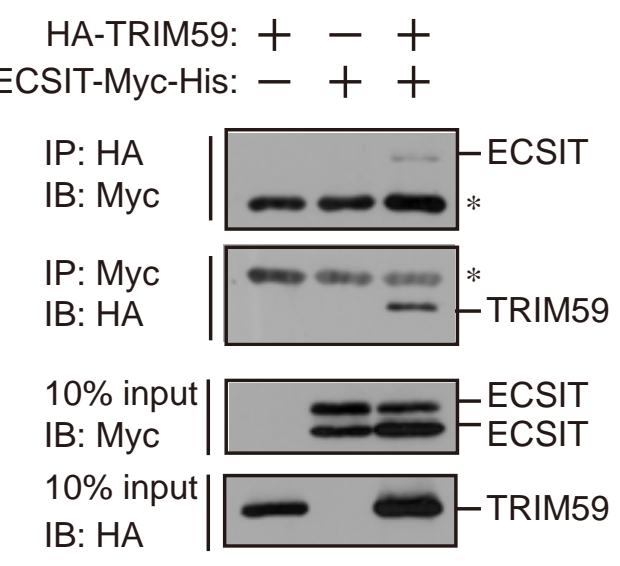

B

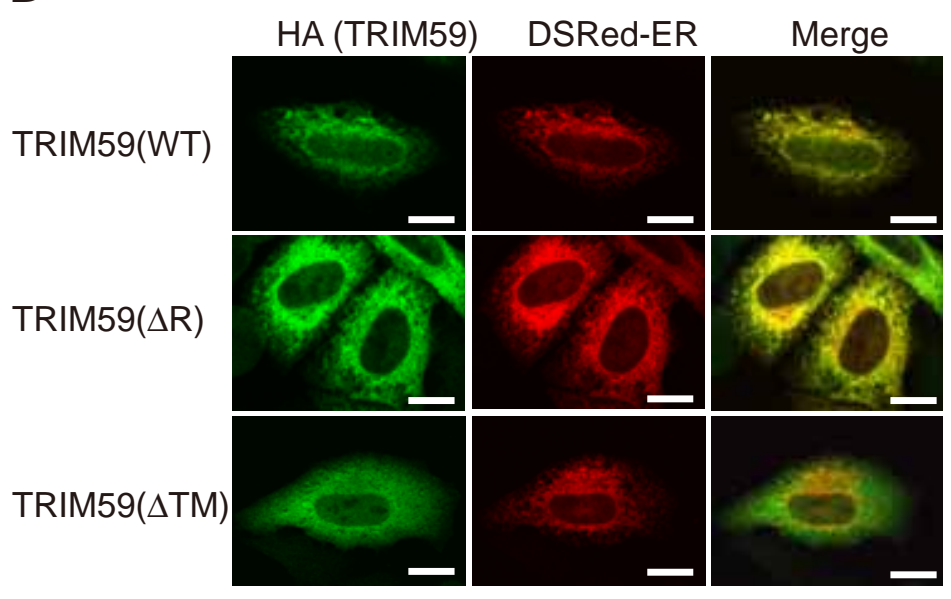

D HA-TRIM59: $\triangle \mathrm{R} \Delta \mathrm{TM}$ B CC $\Delta \mathrm{R} \Delta \mathrm{TM}$ B CC

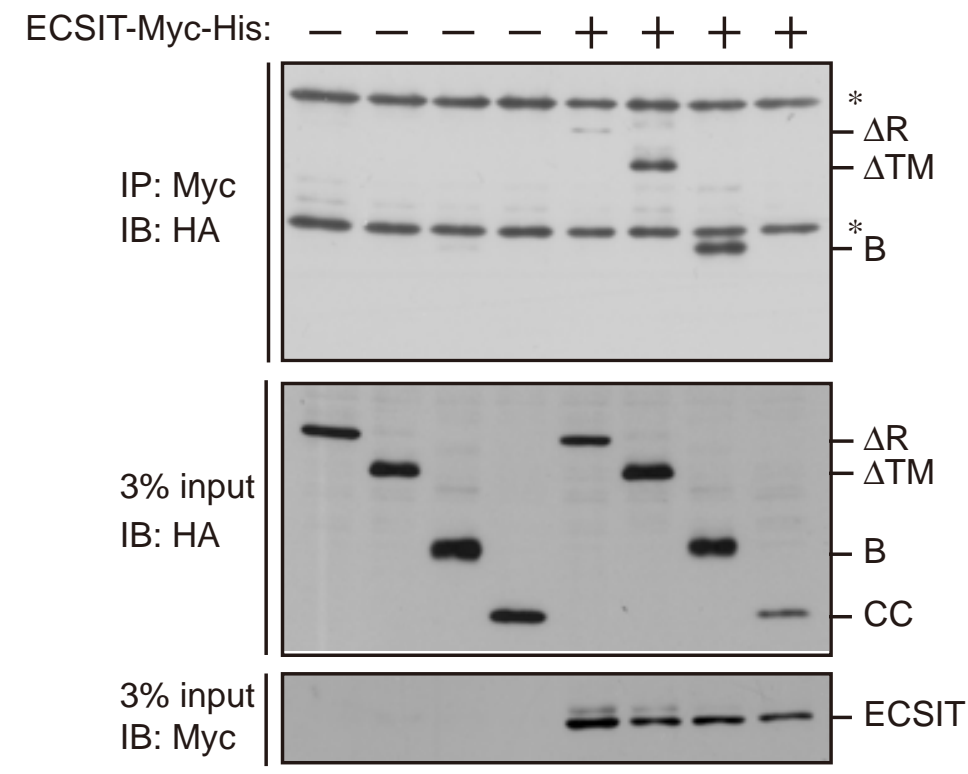

Kondo et al. Fig. 1 
A

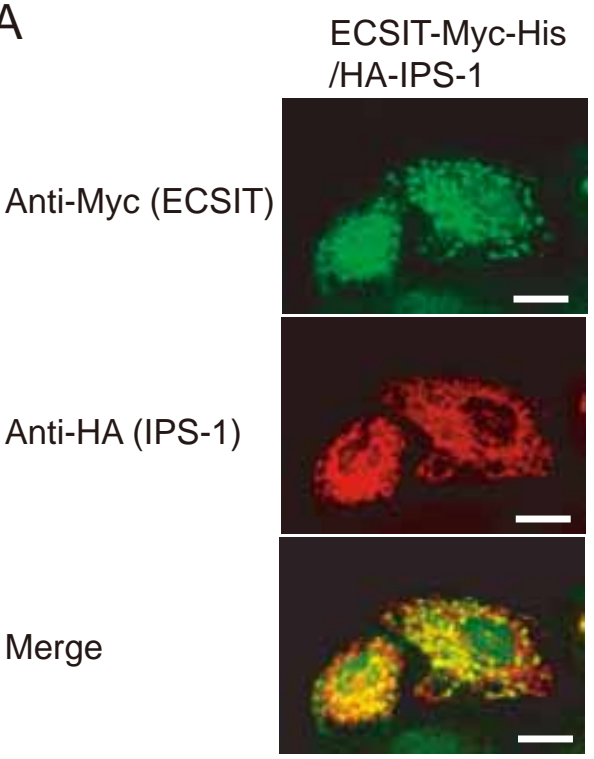

C

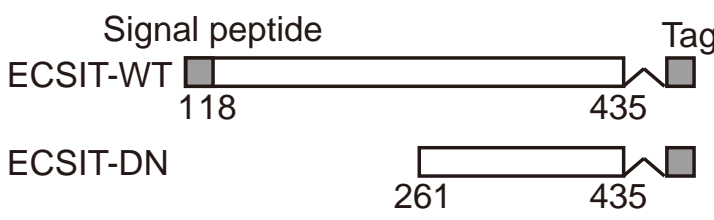

B

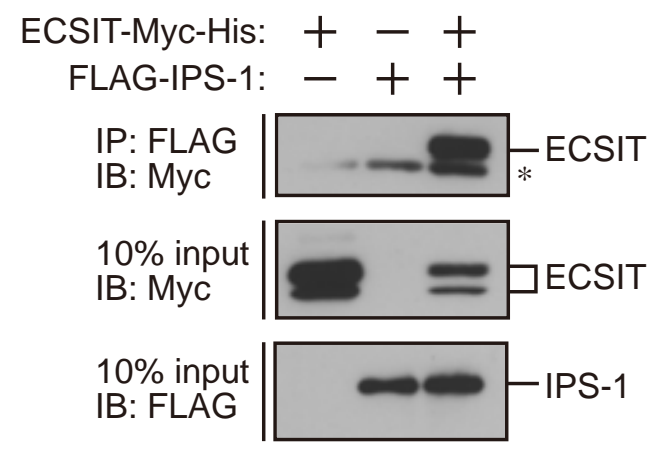

D

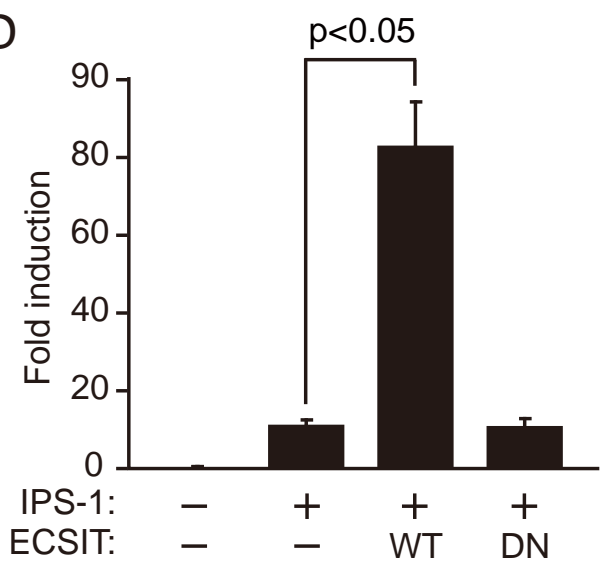

Kondo et al. Fig.2 


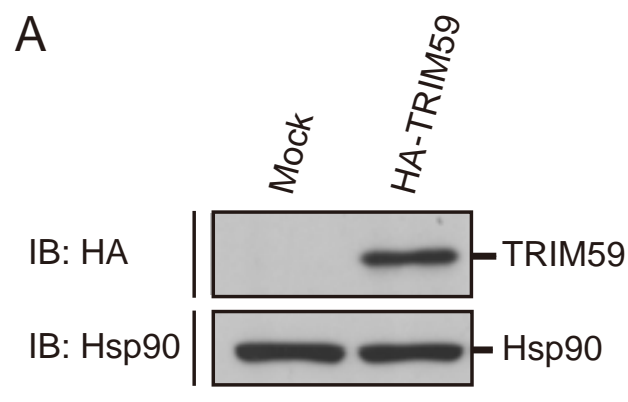

B

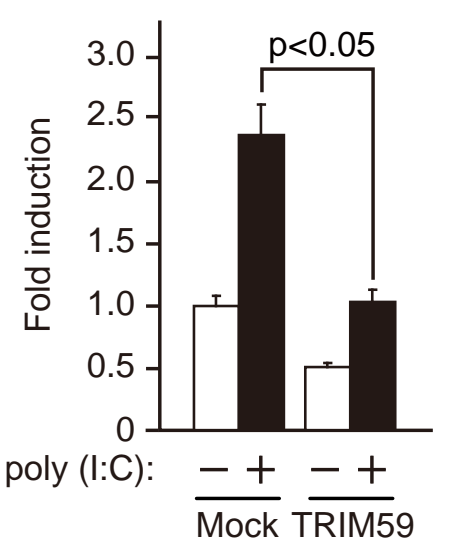

D

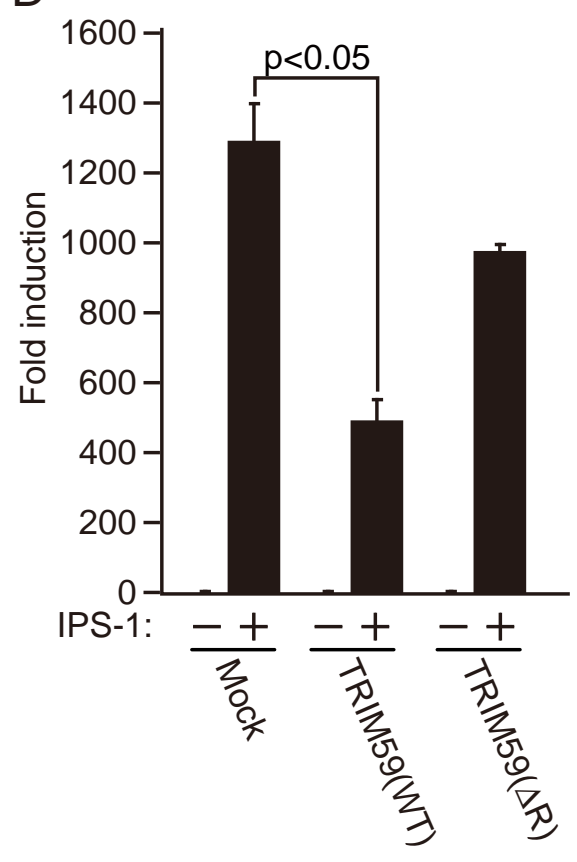

C

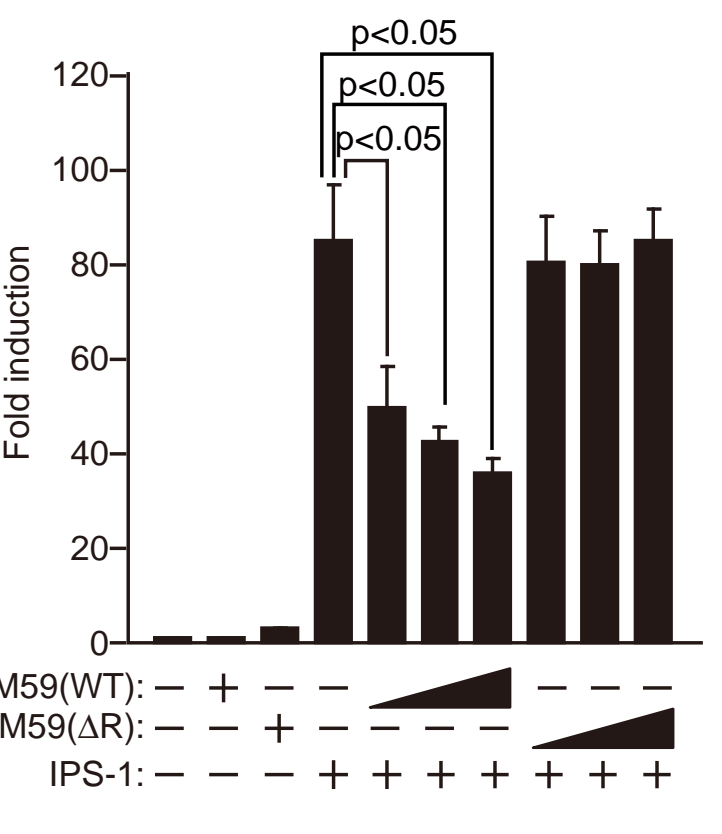

E

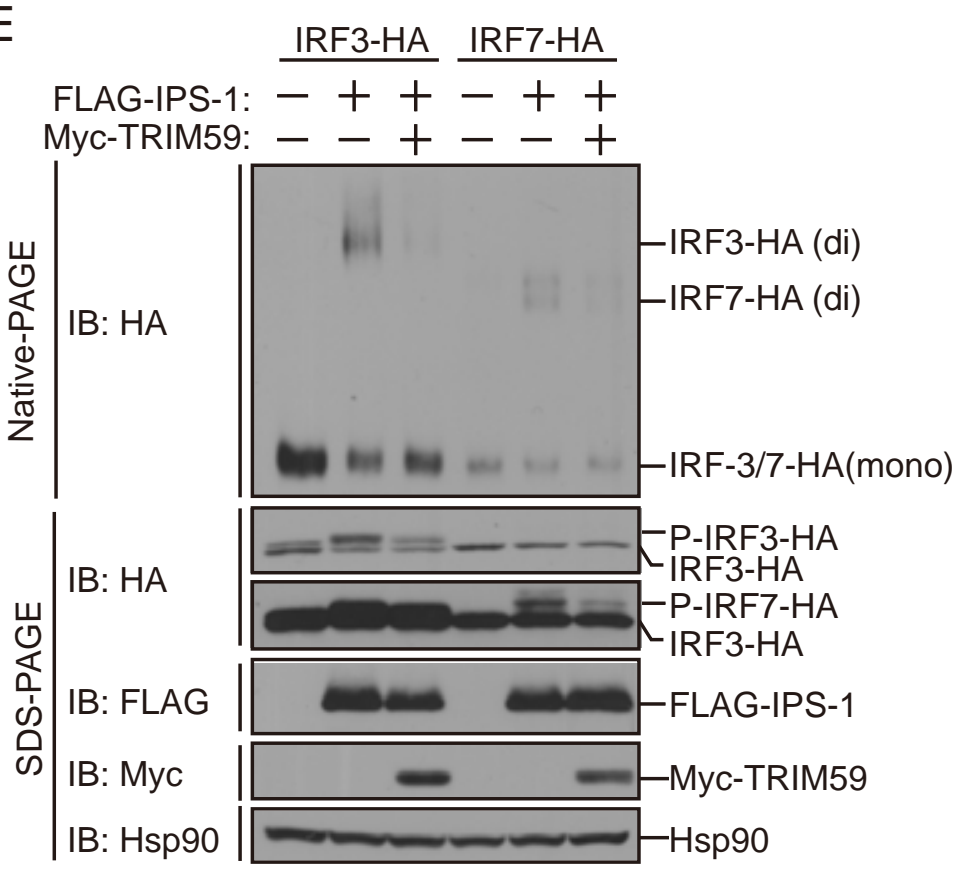

Kondo et al. Fig. 3 

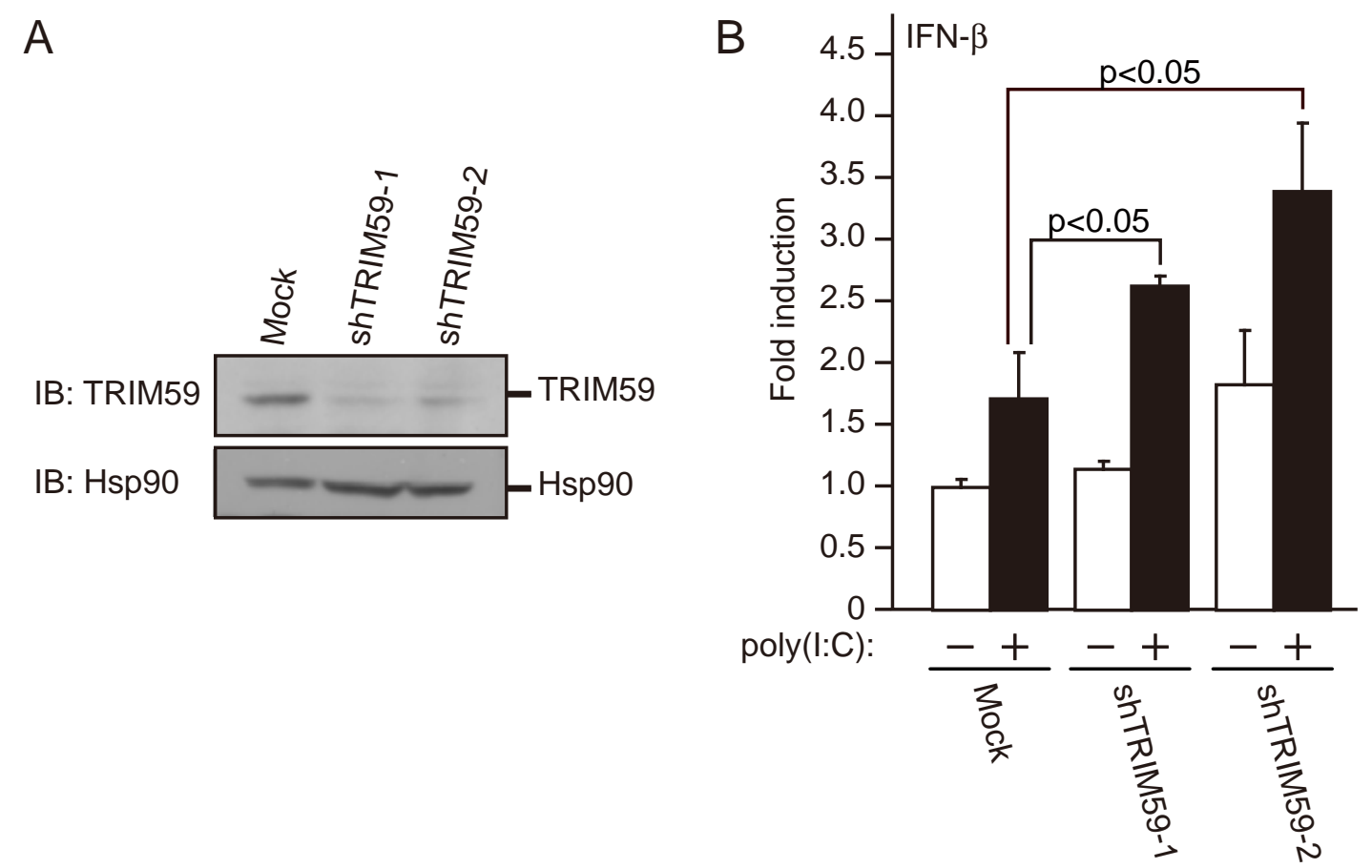

C

D
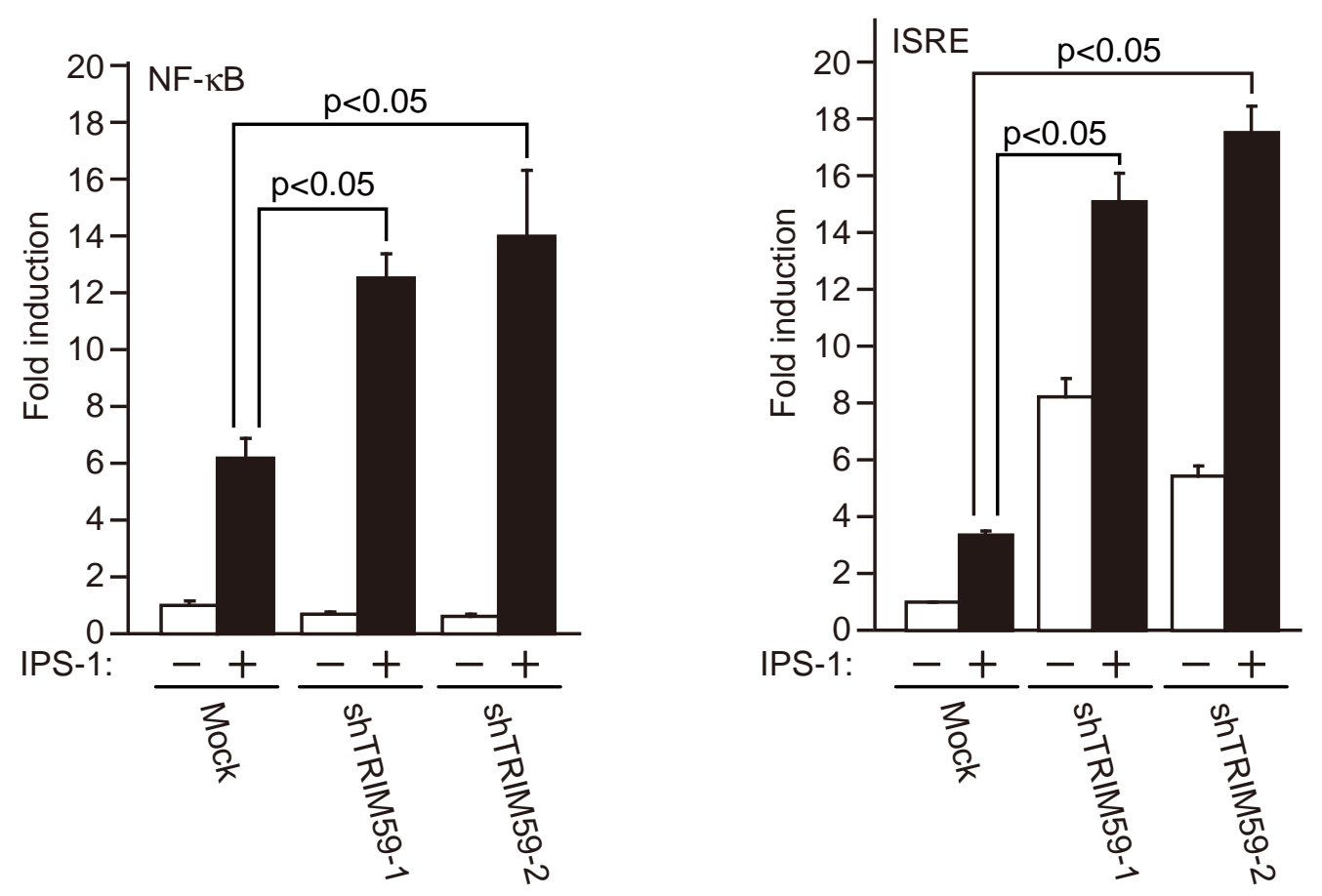

Kondo et al. Fig. 4 